\title{
A multi-component, adaptive Working Memory Assessment Battery (WoMAB): validation and norms in an Italian population sample
}

\author{
Fabrizio Pasotti ${ }^{1}$. Giulia De Luca ${ }^{1}$ Edoardo Nicolò Aiello ${ }^{2,3}$. Chiara Gramegna ${ }^{4} \cdot$ Marco Di Gangi $^{5}$. \\ Giuseppe Foderaro $^{6} \cdot$ Marcello Gallucci $^{4,7} \cdot$ Elena Biglia $^{6} \cdot$ Gabriella Bottini $^{1,7,8}$
}

Received: 26 April 2021 / Accepted: 17 June 2021 / Published online: 29 June 2021

(c) The Author(s) 2021

\begin{abstract}
Background Working memory (WM) abilities are frequently impaired in neurological disorders affecting fronto-parietal cortical/sub-cortical structures. WM deficits negatively influence interventional outcomes and everyday functioning. This study thus aimed at the following: (a) developing and standardizing an ecologically valid task for WM assessment ( Ice Cream Test, ICT); (b) validating and norming a novel WM test (Digit Ordering Test, DOT), as well as providing updated norms for digit span (DS) tasks, in an Italian population sample; (c) introducing a novel scoring procedure for measuring WM. Methods One-hundred and sixty-eight Italian healthy participants-73 male, 95 females; age: $48.4 \pm 19.1$ (18-86); education: $12.1 \pm 4.8$ (4-21) - underwent a thorough WM assessment—DOT, ICT, and both forward and backward DS tasks (FDS, BDS). The ICT requires participants to act as waiters who have to keep track of customers' orders. For each task, WM and total (T) outcomes were computed, i.e., the number of elements in the longest sequence and that of recalled sequences, respectively. Norms were derived via the equivalent score (ES) method.

Results DS ratios (DSRs) were computed for both WM/S and T outcomes on raw DS measures (BDS divided by FDS). Age and education significantly predicted all WM tasks; sex affected FDS and DSR-T scores (males $>$ females). WM measures were highly internally related.

Discussion The present work provides Italian practitioners with a normatively updated, multi-component, adaptive battery for WM assessment (WoMAB) as well as with novel outcomes which capture different WM facets-WM capacity and attentive monitoring abilities.
\end{abstract}

Keywords Working memory $\cdot$ Executive functioning $\cdot$ Normative data $\cdot$ Validation $\cdot$ Ecological validity

Neuropsychological assessment

\section{Introduction}

Working memory (WM) comprises a set of high-order, non-instrumental limited-capacity cognitive functions that allow "temporary storage and manipulation of information necessary for [...] complex cognitive tasks" [2].

The original multi-component model [3] identifies a central executive (CE) component and two modalityspecific sub-components: the phonological loop (PL) and the visuospatial sketchpad (VSS) - processing verbal and visual information, respectively. The CE is a control system of limited capacity that supports complex cognitive

Gabriella Bottini

gabriella.bottini@unipv.it

Extended author information available on the last page of the article activities by suppressing irrelevant information; it allocates attentive resources and allows alternating between different tasks. The PL is a modular subsystem that retains the memory online and prevents it from decaying through both vocal and sub-vocal rehearsals. The VSS temporarily stores and processes visual and spatial information. Most recent formulations [31] introduce a further component, i.e., the episodic buffer-a multi-modal limited-capacity system integrating information from the other components into a unitary episodic representation. WM functioning emerges from the interaction between perceptual and attentive mechanisms and representations stored in the long-term memory system [11, 12].

Converging evidence from neuroimaging and brain injury studies hint at a widespread bilateral fronto-parietal 
both cortical and sub-cortical network being the neural substrate of WM [18, 25, 27, 33].

WM deficits are thus often associated with focal or diffuse brain damages; hence, the neuropsychological assessment constitutes a relevant aspect for cognitive rehabilitation $[13,20,28]$. Several studies have found that WM deficits impair the activities of daily living and affect rehabilitation outcomes [14, 23].

In clinical practice, the digit span (DS) [36] is one of the most widely used tasks to measure the capacity of the auditory-verbal component in WM. According to Baddeley's model [31], the forward (FDS, forward DS) version evaluates the short-term, passive retention of verbal stimuli, whereas the backward version (BDS, backward DS) requires maintaining and actively manipulating information in order to reproduce in reverse order the sequence presented. Both forward and backward versions of the digit span have been validated and normed in Italy $[24,26]$.

Another instrument for assessing auditory-verbal WM is the Digit Ordering Test (DOT) [10, 17, 37], which requires clients to listen to a series of randomly ordered digits and then to recall items in ascending order immediately after their presentation. No Italian standardizations of the DOT are available so far.

Evidence regarding the influence of WM deficits on daily functioning highlights the relevance of ecological validity in cognitive testing [5]. Available tests for the assessment of WM may fail to detect its dysfunction in everyday life [35].

We aimed at developing a composite WM assessment battery (WoMAB) and more specifically at the following: (a) standardizing a novel task that investigates auditoryverbal WM from an ecological perspective; (b) validating and norming the DOT in Italian healthy individuals; and (c) providing updated normative data for DS tasks. A novel scoring procedure will be also proposed: WM and total (T) outcomes, i.e., the number of elements in the longest sequence and that of recalled sequences, respectively. The underlying hypothesis is that WM scores reflect a measure of the auditory-verbal WM capacity, whereas T scores provide insight into attentive monitoring abilities during task execution.

\section{Methods}

\section{Participants}

One-hundred and seventy-three Italian native-speakers individuals were initially recruited from different regions of both Northern and Southern Italy, as well as from the Canton Ticino region of Switzerland. Sample stratification is displayed in Table 1.

Inclusion criteria were as follows: (a) age between 20 and 90 years; (b) years of education between 5 and 18; (c) an adjusted scores on the Mini-Mental State Examination (MMSE) above the established cut-off [21, 22].

Participants were excluded if presenting with neurological disorders, traumatic brain injury, psychiatric disorders, previous brain surgeries, drug abuse, learning disabilities, psychotropic drug treatment, and visual/auditory impairments (participants with corrected-to-normal vision and audition were included).

After applying inclusion/exclusion criteria, $N=168$ individuals were included.

Participants provided written informed consent before being enrolled. The study was approved by the Ethics Committee of the University of Pavia and conducted in accordance with the Declaration of Helsinki.

\section{Materials}

Four auditory-verbal WM tests were administered whose order was counterbalanced across participants to avoid carry-over effects.

Each task started with warming-up trials. Mistakes on preliminary items could be corrected, although without providing execution strategies. Stimuli were pronounced at the rate of one per second, with neutral intonation. Participants were given $15 \mathrm{~s}$ to recall the items. Two lists of the same length were administered; the task was interrupted after two consecutive fails. No cues were provided but self-corrections were accepted. Recalled sequences containing intrusions were scored
Table 1 Sample stratification for age, education, and sex

\begin{tabular}{|c|c|c|c|c|c|c|c|c|}
\hline \multirow[t]{2}{*}{ Education } & & \multicolumn{7}{|l|}{ Age } \\
\hline & & $35 \leq$ & $36-45$ & $46-55$ & $56-65$ & $66-75$ & $76-85$ & $\geq 86$ \\
\hline $5 \leq$ & $\mathrm{M} / \mathrm{F}$ & $0 / 0$ & $0 / 0$ & $0 / 1$ & $3 / 1$ & $4 / 8$ & $4 / 6$ & $0 / 0$ \\
\hline $6-11$ & & $2 / 2$ & $5 / 1$ & $4 / 13$ & $7 / 5$ & $4 / 3$ & $0 / 1$ & $1 / 0$ \\
\hline $12-16$ & & $9 / 11$ & $3 / 4$ & $2 / 6$ & $4 / 1$ & $1 / 1$ & $0 / 1$ & $0 / 0$ \\
\hline$\geq 17$ & & $11 / 21$ & $1 / 0$ & $3 / 3$ & $4 / 6$ & $1 / 0$ & $0 / 0$ & $0 / 0$ \\
\hline
\end{tabular}

Notes: Cells show male/female ratio for each co-occurrence 
as 0 . Both WM and T outcomes were computed for each WM task.

The Ice Cream Test (ICT) is a novel ecologically valid tool investigating auditory-verbal WM. Participants were required to act as if they were waiters in an ice cream shop who have to keep track of customers' orders. Each customer will order a single ice cream flavor; it is required to tell, within $15 \mathrm{~s}$, how many ice creams have to be prepared for each flavor. ICT-WM outcome ranges $0-10$ (longest sequence) and ICT-T 0-16 (recalled sequences).

FDS and BDS tasks were adapted from Monaco et al. [24]. Additionally, the present version introduced one two-digit warming-up sequence. Contrarily to other WM outcomes, that for the FDS was named "Span" (FDS-S) — as not unequivocally targeting WM abilities. FDS-S ranges 0-9 (longest sequence) and FDS-T 0-16 (recalled sequences). BDS-WM ranges 0-8 (longest sequence) and BDS-T 0-14 (recalled sequences).

The DOT [10, 17, 37] consists of presenting a list of randomly ordered digits that have to be recalled in ascending order. DOT-WM outcome ranges 0-8 (longest sequence) and DOT-T 0-12 (recalled sequences).

Test protocols will be provided to interested practitioners upon request to the corresponding author.

\section{Statistical analyses}

By conservatively assuming a small-to-medium size $\left(f^{2}=0.10\right)$ of background predictors effects ( $d$ fnumerator $=3$ ) $[6,24,26]$, the minimum sample size was set at $N=146$ via a power analysis for multiple linear regression analyses [32] (R package $p w r$ ) [9]. $\alpha$ was set at 0.05 and $1-\beta$ at $0.9 ; N$ was yielded by $d$ fnumerator $+d f$ denominator +1 .

Skewness and kurtosis statistics were regarded as suggestive of a violation of the assumption of normality if $>|1|$ and |3|, respectively [19].

Associations of interest between quantitative variables were assessed by means of either Pearson's or Spearman's coefficients. Bonferroni correction for multiple comparisons was applied if adequate.

Norms were drawn by adopting the equivalent score (ES) method [8, 34], a regression-based approach adjusting raw scores (RSs) for significant predictors of interest (or their transforms) and then allotting adjusted scores (ASs) into a 5-level ability scale: $\mathrm{ES}=0$ ( "abnormal"); $\mathrm{ES}=4$ ("highend normal"); $\mathrm{ES}=1,2$, and 3 (respectively, "borderline", "low-end normal", "normal"). Outer and inner tolerance limits (oTL; iTL) were computed to provide an interval estimate for the cut-off (ASs $<$ oTL fall within $\mathrm{ES}=0$ ). Average ESs (AES) [7] were computed for both $\mathrm{T}$ and WM/S outcomes in order to provide a global estimate of attentive monitoring and WM capacity across tasks.

$\mathrm{R}$ 3.6.3 [30] was used for implementing the analyses. Regression studies and calculations of both TLs and ES threshold were implemented as described in Aiello \& Depaoli [1].

\section{Results}

Participants' background features and cognitive scores are summarized in Table 2.

In agreement with Monaco et al.'s [24], the ratios between FDS and BDS tasks were computed (by dividing BDS measures by FDS ones) for both $\mathrm{T}$ al WM/S scores (DSR-T,DSR-WM/S).

Non-derived WM measures were highly internally related $(0.39 \leq r(168) \leq 0.96 ; p<0.001)$-even when adjusting the significance threshold $\left(\alpha_{\text {adjusted }}=0.05 / 28=0.002\right)$.

Ratios were associated with all remaining WM outcomes $(0.23 \leq r(168) \leq 0.72 ; 0.003 \leq p \leq 0.001)$, whereas not with FDS measures.

All WM/S and T measures were negatively associated with age (range: $-0.32 \leq r(168) \leq-0.59 ; p<0.001)$ whereas positively with education (range: $0.2 \leq r(168) \leq 0.46$; $p \leq 0.011)$. Males outperformed females on the BDST, BDS-WM, and DSR-T (range: $2.17 \leq t(166) \leq 2.56$; $0.011 \leq p \leq 0.035$ ); no other sex differences were detected.

When simultaneously tested, age and education transforms revealed to be predictive of DOT (-T and -WM), ICT (-T and $-\mathrm{WM}$ ), and FDS (-T and $-\mathrm{S}$ ) scores (age: range: I.28| $\leq \beta \leq 1.37 \mid$; $|3.03| \leq t \leq 14.22 \mid ; p \leq 0.003$; education: rang $\mathrm{e}:-0.35 \leq \beta \leq-0.24 ;-3.97 \leq t \leq-2.7 ; p \leq 0.008)$. Within a multiple regression model, sex, age, and transformed

Table 2 Participants' background features and WM measures

\begin{tabular}{lll} 
& Background features & \\
$N$ & 168 & \\
Sex (M/F) & $73 / 95$ \\
Age (years) & $48.41 \pm 19.08(18-86)$ & \\
Education (years) & $12.09 \pm 4.8(4-21)$ & \\
MMSE & $28.44 \pm 2.31(17-30)$ & \\
WM measures & $\mathrm{T}$ & WM/S \\
DOT & $7.33 \pm 2.45(1-14)$ & $6.25 \pm 1.48(2-12)$ \\
ICT & $7.29 \pm 2.81(2-16)$ & $6.32 \pm 1.81(3-11)$ \\
FDS & $9.98 \pm 2.48(4-16)$ & $6.45 \pm 1.34(4-9)$ \\
BDS & $7.07 \pm 2.73(2-14)$ & $5.02 \pm 1.5(2-8)$ \\
DSR & $71 \pm .21(.22-1.27)$ & $.78 \pm .18(.18-1.25)$ \\
\hline
\end{tabular}

Notes: $F$, female; $M$, male; $M M S E$, Mini-Mental State Examination; $D O T$, Digit Ordering Test; ICT, Ice Cream Test; $F D S$, forward digit span; FDS, backward digit span; $D S R$, digit span ratio; $T$, Total; $W M$, working memory; $S$, span 
education were found to be predictive of BDS-T (sex: $\beta=-0.21 ; t=-3.48 ; p=0.001 ;$ age: $\beta=-0.47 ; t=-5.93$; $p<0.001$; education: $\beta=-0.21 ; t=-2.71 ; p=0.008)$ and BDS-WM (sex: $\beta=-0.18 ; t=-2.87 ; p=0.005$; age: $\beta=-0.46 ; t=-5.61 ; p<0.001$; education: $\beta=-0.19$; $t=-2.38 ; p=0.018)$. With respect to ratios, sex and transformed age were found to be predictive of DSR-T scores (sex: $\beta=-0.08 ; t=-2.49 ; p=0.014$; age: $\beta=-0.16$; $t=-4.6 ; p<0.001)$, whereas only inverse age significantly predicted DSR-WM/S scores $(\beta=4.08 ; t=3.39 ; p=0.001)$.

The mean AES scores were $3.06 \pm 0.7(0.6-4)$ and $3.11 \pm 0.7(0.6-4)$ for WM and T outcomes, respectively. No association with either age or education was found with respect to both AESs. However, AES-T was significantly higher $(t(166)=2.4 ; p=0.018)$ for males than for males.
Correction coefficients for selected co-occurrences of background predictors along with equations for adjusting RSs are reported in Table 3 (DOT and ICT), Table 4 (FDS and BDS), and Table 5 (DSR). Normative values for all measures are reported in Table 6. For AESs, only TLS are provided [7].

\section{Discussion}

This work provides Italian neuropsychologists with a novel standardized tool for the ecological assessment of auditoryverbal WM abilities (ICT), as well as with norms and validity evidence for the DOT. Both ICT and DOT measures proved to converge with widespread WM measures (FDS and BDS).
Table 3 Adjustment grids according to age and education for Digit Ordering Test (DOT) and Ice Cream Test (ICT) total (T) and working memory (WM) raw scores

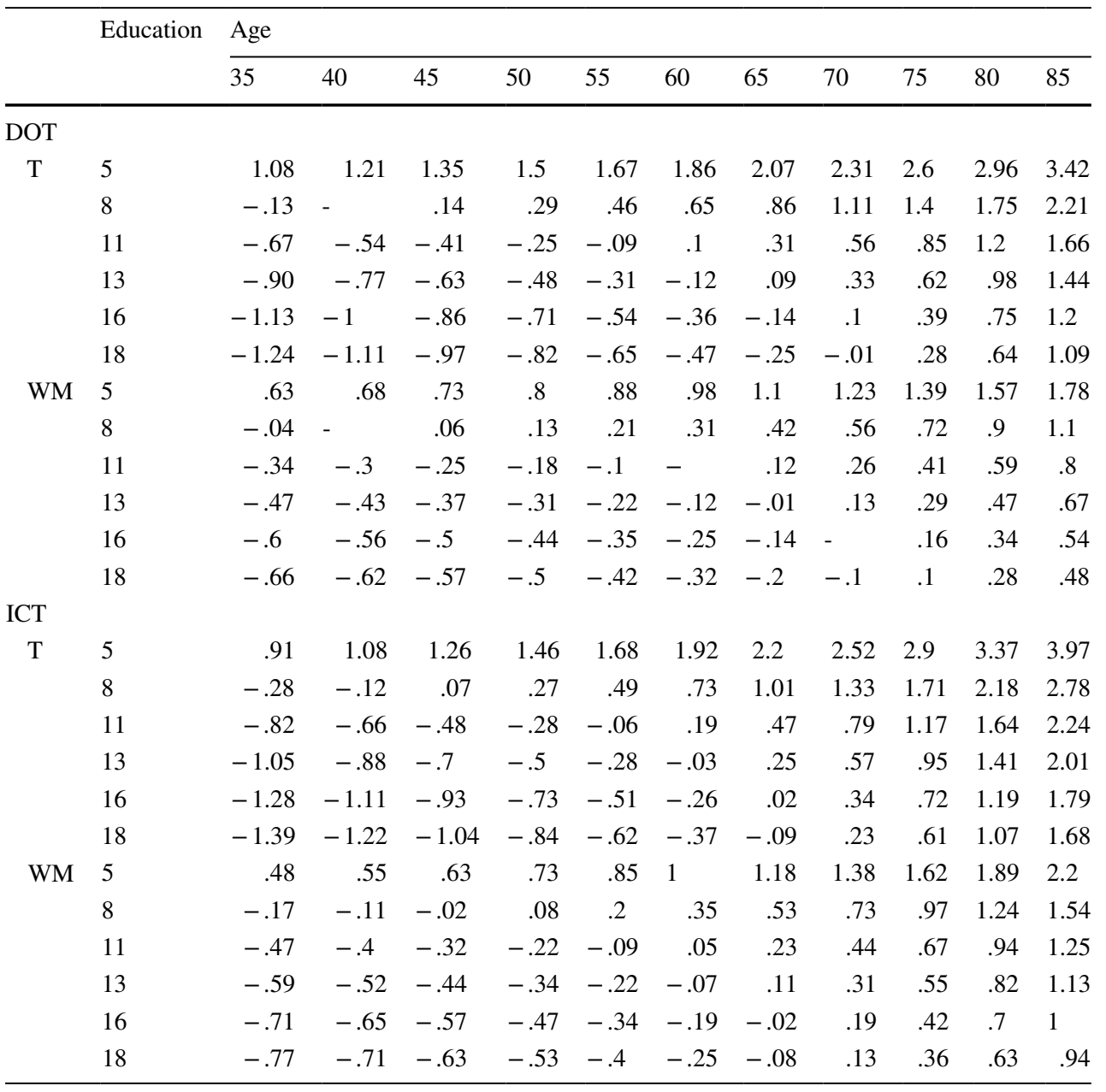

DOT-T adjusted score $=$ raw score $-1.590625 *[\ln (100-$ age $)-3.862482]+16.07802 *$ [(1/education $)-.101788]$. DOT-WM adjusted $s c o r e=$ raw score $+.000002 *\left[\left(\right.\right.$ age $\left.\left.^{\wedge} 3\right)-166,465.803571\right]+8.9739$ $*$ [(1/education $)-.101788]$. ICT-T adjusted score $=$ raw score $-2.087459 *[\ln (100-$ age $)-3.862482]+1$ $5.88892 *[(1 /$ education $)-.101788]$. ICT-WM adjusted score $=$ raw score $+.000003 *\left[\left(\right.\right.$ age $\left.^{\wedge} 3\right)-166,465$ $.803571]+8.691697 *[(1 /$ education $)-.101788]$. Significant decimals of adjustment factors are displayed. Adjustment factors have been extracted from the aforementioned formula and do not always reflect empirical co-occurrence 
Table 4 Adjustment grids according to age and education for forward and backward digit span (F/BDS) total (T) and span/ working memory (S/WM) raw scores

\begin{tabular}{|c|c|c|c|c|c|c|c|c|c|c|c|c|c|}
\hline & \multicolumn{2}{|c|}{ Education } & \multicolumn{11}{|l|}{ Age } \\
\hline & & & 35 & 40 & 45 & 50 & 55 & 60 & 65 & 70 & 75 & 80 & 85 \\
\hline \multicolumn{14}{|l|}{ FDS } \\
\hline \multirow[t]{6}{*}{$\mathrm{T}$} & & 5 & .6 & .77 & .95 & 1.15 & 1.37 & 1.61 & 1.89 & 2.21 & 2.59 & 3.05 & 3.65 \\
\hline & & 8 & -.35 & -.19 & -.01 & .19 & .41 & .66 & .93 & 1.26 & 1.64 & 2.1 & 2.7 \\
\hline & & 11 & -.79 & -.62 & -.44 & -.24 & -.02 & .22 & .5 & .82 & 1.2 & 1.67 & 2.27 \\
\hline & & 13 & -.97 & -.8 & -.62 & -.42 & -.2 & .05 & .32 & .64 & 1.02 & 1.49 & 2.09 \\
\hline & & 16 & -1.15 & -.98 & -.8 & -.6 & -.38 & -.14 & .14 & .46 & .84 & 1.31 & 1.9 \\
\hline & & 18 & -1.24 & -1.07 & -.89 & -.69 & -.47 & -.23 & .05 & .37 & .75 & 1.22 & 1.82 \\
\hline \multirow[t]{6}{*}{ S } & & 5 & .23 & .29 & .37 & .47 & 6 & .75 & .92 & 1.13 & 1.37 & 1.64 & 1.94 \\
\hline & & 8 & -.23 & -.17 & -.08 & .02 & .14 & .29 & .47 & .67 & .91 & 1.18 & 1.48 \\
\hline & & 11 & -.44 & -.37 & -.29 & -.19 & -.07 & .08 & .26 & .46 & .7 & .97 & 1.28 \\
\hline & & 13 & -.52 & -.46 & -.38 & -.28 & -.15 & - & .17 & .38 & .61 & .88 & 1.19 \\
\hline & & 16 & -.61 & -.55 & -.47 & -.36 & -.24 & -.09 & .08 & .29 & .53 & .8 & 1.1 \\
\hline & & 18 & -.65 & -.59 & -.51 & -.41 & -.28 & -.13 & .04 & .25 & .48 & .75 & 1.06 \\
\hline \multicolumn{14}{|l|}{ BDS } \\
\hline \multirow[t]{12}{*}{$\mathrm{T}$} & $\hat{0}$ & 5 & -.39 & -.06 & .26 & .59 & .92 & 1.25 & 1.58 & 1.91 & 2.24 & 2.56 & 2.89 \\
\hline & & 8 & -1.21 & -.89 & -.56 & -.23 & .1 & .43 & .76 & 1.09 & 1.41 & 1.74 & 2.07 \\
\hline & & 11 & -1.59 & -1.26 & -.93 & -.6 & -.27 & .06 & .38 & .71 & 1.04 & 1.37 & 1.7 \\
\hline & & 13 & -1.74 & -1.41 & -1.08 & -.76 & -.43 & -.1 & .23 & .56 & .89 & 1.22 & 1.54 \\
\hline & & 16 & -1.9 & -1.57 & -1.24 & -.91 & -.58 & -.26 & .07 & .4 & .73 & 1.06 & 1.39 \\
\hline & & 18 & -1.97 & -1.65 & -1.32 & -.99 & -.66 & -.33 & - & .32 & .65 & .98 & 1.31 \\
\hline & 우 & 5 & .78 & 1.11 & 1.44 & 1.77 & 2.1 & 2.42 & 2.75 & 3.08 & 3.41 & 3.74 & 4.07 \\
\hline & & 8 & -.04 & .29 & .62 & .95 & 1.27 & 1.6 & 1.93 & 2.26 & 2.59 & 2.92 & 3.24 \\
\hline & & 11 & -.41 & -.08 & .24 & .57 & .9 & 1.23 & 1.56 & 1.89 & 2.21 & 2.54 & 2.87 \\
\hline & & 13 & -.57 & -.24 & .09 & .42 & .75 & 1.08 & 1.4 & 1.73 & 2.06 & 2.39 & 2.72 \\
\hline & & 16 & -.72 & -.4 & -.07 & .26 & .59 & .92 & 1.25 & 1.58 & 1.9 & 2.23 & 2.56 \\
\hline & & 18 & -.8 & -.47 & -.14 & .19 & .51 & .84 & 1.17 & 1.5 & 1.83 & 2.16 & 2.48 \\
\hline \multirow[t]{12}{*}{ WM } & 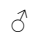 & 5 & -.21 & -.04 & .14 & .32 & .49 & .67 & .84 & 1.02 & 1.2 & 1.37 & 1.55 \\
\hline & & 8 & -.62 & -.45 & -.27 & -.1 & .08 & .26 & .43 & .61 & .79 & .96 & 1.14 \\
\hline & & 11 & -.81 & -.63 & -.46 & -.28 & -.11 & .07 & .25 & .42 & .6 & .78 & .95 \\
\hline & & 13 & -.89 & -.71 & -.53 & -.36 & -.18 & -.01 & .17 & .35 & .52 & .7 & .88 \\
\hline & & 16 & -.97 & -.79 & -.61 & -.44 & -.26 & -.08 & .09 & .27 & .44 & .62 & .8 \\
\hline & & 18 & -1 & -.83 & -.65 & -.48 & -.3 & -.12 & .05 & .23 & .41 & .58 & .76 \\
\hline & 우 & 5 & .34 & .52 & .7 & .87 & 1.05 & 1.22 & 1.4 & 1.58 & 1.75 & 1.93 & 2.11 \\
\hline & & 8 & -.07 & .11 & .29 & .46 & .64 & .81 & .99 & 1.17 & 1.34 & 1.52 & 1.7 \\
\hline & & 11 & -.25 & -.08 & .1 & .27 & .45 & .63 & .8 & .98 & 1.16 & 1.33 & 1.51 \\
\hline & & 13 & -.33 & -.15 & .02 & .2 & .37 & .55 & .73 & .9 & 1.08 & 1.26 & 1.43 \\
\hline & & 16 & -.41 & -.23 & -.06 & .12 & .3 & .47 & .65 & .82 & 1 & 1.18 & 1.35 \\
\hline & & 18 & -.45 & -.27 & -.09 & .08 & .26 & .43 & .61 & .79 & .96 & 1.14 & 1.32 \\
\hline
\end{tabular}

Notes: $M$, male; $F$, female; FDS-T-adjusted score $=$ raw score $-2.082331 *[\ln (100-$ age $)-3.862482]+12.7$ $1517 *[(1 /$ education $)-.101788]$. FDS-S adjusted score $=$ raw score $+.000003 *\left[\left(\right.\right.$ age $\left.\left.{ }^{\wedge} 3\right)-166,465.803571\right]$ $+6.09889 *[(1 /$ education $)-.101788]$. BDS-T adjusted score $=$ raw score $+.065689 *($ age -48.41071$)+10.9$ $5221 *[(1 /$ education $)-.101788]+.587136$ if female; -.587136 if male. BDS-WM adjusted score $=$ raw scor $\mathrm{e}+.035249 *($ age -48.41071$)+5.473036 *[(1 /$ education $)-.101788]+.278456$ if female; -.278456 if male. Significant decimals of adjustment factors are displayed. Adjustment factors have been extracted from the aforementioned formula and do not always reflect empirical co-occurrences
Moreover, updated normative data are provided for both the FDS and the DBS. Cut-offs here reported are substantially comparable to those found by Monaco et al. [24] (FDS: 2.78 vs. 2.65 ,BDS: 4.46 vs. 4.26 ). Inconsistently with previous normative studies [24, 26], as well as with contributions on sex differences in WM abilities [16], males scored higher than females on BDS and DSR measures. The present findings thus counterbalance those 
Table 5 Adjustment grids according to age, education, and sex for the backward digit span (BDS) total (T) and Working Memory (WM) raw scores

\begin{tabular}{|c|c|c|c|c|c|c|c|c|c|c|c|c|}
\hline \multirow[t]{2}{*}{ DSR } & & \multicolumn{11}{|l|}{ Age } \\
\hline & & 35 & 40 & 45 & 50 & 55 & 60 & 65 & 70 & 75 & 80 & 85 \\
\hline \multirow[t]{2}{*}{$\mathrm{T}$} & 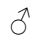 & -.074 & -.053 & -.035 & -.018 & -.003 & .01 & .023 & .034 & .045 & .055 & .065 \\
\hline & q & - & .021 & .04 & .056 & .071 & .085 & .097 & .109 & .12 & .13 & .139 \\
\hline WM/S & & -.015 & - & .011 & .02 & .027 & .034 & .039 & .043 & .047 & .051 & .054 \\
\hline
\end{tabular}

Notes. DSR-T adjusted score $=$ raw score $+.156681 *(\ln ($ age $)-3.791483)-.037286$ if $\mathrm{M}+.037286$ if $\mathrm{F}$. DSR-WM/S adjusted score $=$ raw score $-4.081518 *((1 /$ age $)-.024893)$. Significant decimals of adjustment factors are displayed. Adjustment factors have been extracted from the aforementioned formula and do not always reflect empirical co-occurrences hinting at a prominent male-female difference being detectable on visuospatial but not in phonological WM tasks [29].

It is also worth noting that the DSR in the present study differs from that of Monaco et al. [24] since it was computed on raw rather than adjusted scores.

This work also introduced a novel scoring procedure that provides insights about different facets of phonological WM-WM capacity (WM/S) and attentive monitoring abilities during task execution $(\mathrm{T})[4,15]$.

AESs here reported further contribute to the adaptive nature of this composite battery. Indeed, the WoMAB allows an in-depth profiling of WM abilities by yielding both single-task-level (ESs) and global (AESs) standardized scores with respect to considered outcomes ( $\mathrm{T}$ and $\mathrm{WM} / \mathrm{S})$. Although both AESs proved to be independent of age and education [7], practitioners should nonetheless exert caution when interpreting AES-T measures due to sex differences.

A limitation has to be finally acknowledged regarding sample stratification: certain co-occurrences of age and education levels indeed happened to be poorly represented (e.g., highly educated individuals aged $\geq 75$ years) — possibly due to sampling biases. This should lead to exercising attention when adjusting RSs of individuals with these background features. However, it is believed that the soundness of regression analyses as far as statistical power is concerned allows sufficiently adequate predictions of adjustment factors for the aforementioned co-occurrences too.

In conclusion, this study validated and normed the WoMAB, a multi-component, flexible battery for WM assessment in adult neurological populations. Its novel scoring procedure allows assessing both WM capacity (longest sequence) and task-related attentive processes (number of recalled sequences). Moreover, the WoMAB encompasses ecologically valid measures that can help practitioners evaluate the impact of WM deficits in patients' daily activities.

Acknowledgements The authors are grateful to all participants.

Funding Open access funding provided by Università degli Studi di Pavia within the CRUI-CARE Agreement.
Table 6 Equivalent scores for DOT, ICT, FDS, BDS and RDS adjusted scores

\begin{tabular}{|c|c|c|c|c|c|c|c|}
\hline & \multirow[t]{2}{*}{ oTL } & \multirow[t]{2}{*}{ iTL } & \multicolumn{5}{|c|}{ Equivalent scores } \\
\hline & & & 0 & 1 & 2 & 3 & 4 \\
\hline DOT-T & 3.27 & 4.72 & $\leq 3.27$ & $3.28-4.72$ & $4.73-5.76$ & $5.77-7.35$ & $\geq 7.36$ \\
\hline DOT-WM & 4.05 & 4.5 & $\leq 4.05$ & 4.064 .57 & $4.58-5.42$ & $5.43-6.31$ & $\geq 6.32$ \\
\hline ICT-T & 2.48 & 3.7 & $\leq 2.48$ & $2.49-4.14$ & $4.15-5.7$ & $5.71-7.03$ & $\geq 7.04$ \\
\hline ICT-WM & 3.69 & 4.17 & $\leq 3.69$ & $3.7-4.22$ & $4.23-5.19$ & $5.2-6.19$ & $\geq 6.2$ \\
\hline FDS-T & 6.42 & 7.35 & $\leq 6.42$ & $6.43-7.4$ & $7.41-8.49$ & $8.5-9.88$ & $\geq 9.89$ \\
\hline FDS-S & 4.45 & 5.17 & $\leq 4.45$ & $4.46-5.22$ & $5.23-5.58$ & $5.59-6.31$ & $\geq 6.32$ \\
\hline BDS-T & 3.35 & 4.26 & $\leq 3.35$ & $3.36-4.38$ & $4.39-5.67$ & $5.68-7.16$ & $\geq 7.17$ \\
\hline BDS-WM & 2.77 & 3.41 & $\leq 2.77$ & $2.78-3.45$ & $3.46-4.26$ & $4.27-4.88$ & $\geq 4.89$ \\
\hline DSR-T & .393 & .486 & $\leq .393$ & $.394-.502$ & $.503-.638$ & $.639-.735$ & $\geq .736$ \\
\hline DSR-WM/S & .461 & .541 & $\leq .461$ & $.462-.55$ & $.551-.659$ & $.66-.786$ & $\geq .787$ \\
\hline AES-T & 1.4 & 2.2 & $\leq .1 .4$ & - & - & - & - \\
\hline AES-WM/S & 1.2 & 2.2 & $\leq .1 .2$ & - & - & - & - \\
\hline
\end{tabular}

$\mathrm{T}=$ total; $\quad$ WM=Working Memory; $\mathrm{S}=\mathrm{Span} ; \mathrm{DOT}=$ Digit Ordering Test; ICT=Icre Cream Test; FDS=Forward Digit Span; BDS=Backward Digit Span; DSR=Digit Span Ratio; AES=average Equivalent Score; oTL $=$ outer tolerance limit; $\mathrm{iTL}=$ inner tolerance limit. 


\section{Declarations}

Conflict of interest The authors declare no competing interests.

Ethical approval Participants provided informed consent before being enrolled. The study was approved by the Ethics Committee of the University of Pavia.

Open Access This article is licensed under a Creative Commons Attribution 4.0 International License, which permits use, sharing, adaptation, distribution and reproduction in any medium or format, as long as you give appropriate credit to the original author(s) and the source, provide a link to the Creative Commons licence, and indicate if changes were made. The images or other third party material in this article are included in the article's Creative Commons licence, unless indicated otherwise in a credit line to the material. If material is not included in the article's Creative Commons licence and your intended use is not permitted by statutory regulation or exceeds the permitted use, you will need to obtain permission directly from the copyright holder. To view a copy of this licence, visit http://creativecommons.org/licenses/by/4.0/.

\section{References}

1. Aiello EN, Depaoli EG (2021) Norms and standardizations in neuropsychology via equivalent scores: software solutions and practical guides. Neurol Sci. https://doi.org/10.1007/ s10072-021-05374-0

2. Baddeley A (1992) Working memory. Science 255:556-559

3. Baddeley AD, Hitch G (1974) Working memory. In Psychology of learning and motivation. Academic Press, pp 47-89

4. Bao M, Li ZH, Chen XC, Zhang DR (2006) Backward inhibition in a task of switching attention within verbal working memory. Brain Res Bull 69(2):214-221

5. Bianchi A (2013) L'esame neuropsicologico dell'adulto. Giunti Psychometrics, Firenze

6. Brugnolo A, De Carli F, Accardo J, Amore M, Bosia LE, Bruzzaniti C, Cappa SF, Cocito L, Colazzo G, Ferrara M, Ghio L (2016) An updated Italian normative dataset for the Stroop color word test (SCWT). Neurol Sci 37:365-372

7. Capitani E, Laiacona M (1997) Composite neuropsychological batteries and demographic correction: standardization based on equivalent scores, with a review of published data. J Clin Exp Neuropsychol 19:795-809

8. Capitani E, Laiacona M (2017) Outer and inner tolerance limits: their usefulness for the construction of norms and the standardization of neuropsychological tests. Clin Neuropsychol 31:1219-1230

9. Champely S (2020) pwr: basic functions for power analysis (R package version 1.3-0) [Computer software]. The Comprehensive R Archive Network. Available from https://CRAN.R-proje ct.org/package $=$ pwr. Accessed 15 March 2021

10. Cooper JA, Sagar HJ, Jordan N, Harvey NS, Sullivan EV (1991) Cognitive impairment in early, untreated Parkinson's disease and its relationship to motor disability. Brain 114:2095-2122

11. D'Esposito M, Postle BR (2015) The cognitive neuroscience of working memory. Annu Rev Psychol 66:115-142

12. Eriksson J, Vogel EK, Lansner A, Bergström F, Nyberg L (2015) Neurocognitive architecture of working memory. Neuron 88:33-46

13. Ferber S, Ruppel J, Danckert J (2020) Visual working memory deficits following right brain damage. Brain Cogn 142:105566
14. Fitri FI, Fithrie A, Rambe AS (2020) Association between working memory impairment and activities of daily living in poststroke patients. Med Glas 17:433-438

15. Fukuda K, Woodman GF, Vogel EK (2015) Individual differences in visual working memory capacity: contributions of attentional control to storage. Mechanisms of sensory working memory: Attention and perfomance XXV, 105

16. Harness A, Jacot L, Scherf S, White A, Warnick JE (2008) Sex differences in working memory. Psychol Rep 103(1):214-218

17. Hoppe C, Müller U, Werheid K, Thöne A, von Cramon YD (2000) Digit Ordering Test: clinical, psychometric, and experimental evaluation of a verbal working memory test. Clin Neuropsychol 14:38-55

18. Jo JM, Kim YH, Ko MH, Ohn SH, Joen B, Lee KH (2009) Enhancing the working memory of stroke patients using tDCS. Am J Phys Med Rehabil 88:404-409

19. Kim HY (2013) Statistical notes for clinical researchers: assessing normal distribution (2) using skewness and kurtosis. Restor Dent Endod 38:52-54

20. Lugtmeijer S, Lammers NA, de Haan EH, de Leeuw FE, Kessels RP (2020) Post-stroke working memory dysfunction: a metaanalysis and systematic review. Neuropsychol Rev 1-18

21. Magni E, Binetti G, Bianchetti A, Rozzini R, Trabucchi M (1996) Mini-Mental State Examination: a normative study in Italialan elderly population. Eur J Neurol 3:198-202

22. Measso G, Cavarzeran F, Zappala G, Lebowitz BD, Crook TH, Pirozzolo FJ, Amaducci LA, Massari D, Grigoletto F (1993) The Mini-Mental State Examination: normative study of an Italian random sample. Dev Neuropsychol 9:77-85

23. Mellon L, Brewer L, Hall P, Horgan F, Williams D, Hickey A (2015) Cognitive impairment six months after ischaemic stroke: a profile from the ASPIRE-S study. BMC Neurol 15:1-9

24. Monaco M, Costa A, Caltagirone C, Carlesimo GA (2013) Forward and backward span for verbal and visuo-spatial data: standardization and normative data from an Italian adult population. Neurol Sci 34:749-754

25. Müller NG, Knight RT (2006) The functional neuroanatomy of working memory: contributions of human brain lesion studies. Neuroscience 139:51-58

26. Orsini A, Grossi D, Capitani E, Laiacona M, Papagno C, Vallar G (1987) Verbal and spatial immediate memory span: normative data from 1355 adults and 1112 children. Italian J Neurol Sci 8:537-548

27. Paulesu E, Frith CD, Frackowiak RS (1993) The neural correlates of the verbal component of working memory. Nature 362(6418):342-345

28. Peterburs J, Bellebaum C, Koch B, Schwarz M, Daum I (2010) Working memory and verbal fluency deficits following cerebellar lesions: relation to interindividual differences in patient variables. The Cerebellum 9:375-383

29. Piccardi L, D’Antuono G, Marin D, Boccia M, Ciurli P, Incoccia C, Antonucci G, Verde P, Guariglia C (2019) New evidence for gender differences in performing the Corsi Test but not the Digit Span: Data from 208 individuals. Psychol Stud 64:411-419

30. R Core Team (2019) R: a language and environment for statistical computing. (Version 3.6.3) [Computer software]. Retrieved from https://cran.r-project.org. Accessed 15 March 2021

31. Repovš G, Baddeley A (2006) The multi-component model of working memory: explorations in experimental cognitive psychology. Neuroscience 139:5-21

32. Rothstein HR, Borenstein M, Cohen J, Pollack S (1990) Statistical power analysis for multiple regression/correlation: a computer program. Educ Psychol Measur 50:819-830

33. Rottschy C, Langner R, Dogan I, Reetz K, Laird AR, Schulz JB, Fox PT, Eickhoff SB (2012) Modelling neural correlates of 
working memory: a coordinate-based meta-analysis. Neuroimage 60:830-846

34. Spinnler H, Tognoni G (1987) Standardizzazione e taratura italiana di test neuropsicologici. Ital J Neurol Sci 6:1-120

35. Vallat-Azouvi C, Pradat-Diehl P, Azouvi P (2012) The Working Memory Questionnaire: a scale to assess everyday life problems related to deficits of working memory in brain injured patients. Neuropsychol Rehabil 22:634-649
36. Wechsler D (1939) Wechsler-Bellevue Intelligence Scale. The Psychological Corporation, New York

37. Werheid K, Hoppe C, Thöne A, Müller U, Müngersdorf M, von Cramon DY (2002) The adaptive Digit Ordering Test clinical application, reliability, and validity of a verbal working memory test. Arch Clin Neuropsychol 17:547-565

Publisher's note Springer Nature remains neutral with regard to jurisdictional claims in published maps and institutional affiliations.

\section{Authors and Affiliations}

\section{Fabrizio Pasotti ${ }^{1}$ Giulia De Luca ${ }^{1}$ Edoardo Nicolò Aiello ${ }^{2,3}$. Chiara Gramegna ${ }^{4}$ - Marco Di Gangi ${ }^{5}$. Giuseppe Foderaro $^{6} \cdot$ Marcello Gallucci $^{4,7} \cdot$ Elena Biglia $^{6} \cdot$ Gabriella Bottini $^{1,7,8}$ (D)}

Fabrizio Pasotti

fabriziopasotti@gmail.com

Giulia De Luca

giulia.deluca.psy@gmail.com

Edoardo Nicolò Aiello

e.aiello5@campus.unimib.it

Chiara Gramegna

c.gramegna@campus.unimib.it

Marco Di Gangi

m.digangi@digangivicini.ch

Giuseppe Foderaro

giuseppe.foderaro@eoc.ch

Marcello Gallucci

marcello.gallucci@unimib.it

Elena Biglia

elena.biglia@eoc.ch
1 Department of Brain and Behavioural Sciences, University of Pavia, Pavia, Italy

2 School of Medicine and Surgery, University of Milano-Bicocca, Monza, Italy

$3 \mathrm{PhD}$ in Neuroscience, University of Milano-Bicocca, Monza, Italy

4 Department of Psychology, University of Milano-Bicocca, Milano, Italy

5 Studio Di Gangi \& Vicini Psicologia Psicoterapia e Neuropsicologia, Minusio, Switzerland

6 Neurocentro Della Svizzera Italiana, Ente Ospedaliero Cantonale Ospedale Regionale Di Lugano, Lugano, Switzerland

7 Milan Center for Neuroscience (NeuroMI), Milano, Italy

8 Cognitive Neuropsychology Centre, ASST Grande Ospedale Metropolitano Niguarda, Milan, Italy 